\title{
Immediate implants supporting single crown restoration: a 5-year clinical study.
}

IMPLANT THERAPY OUTCOMES, SURGICAL ASPECTS
España A, Jiménez A, Garrido N, Ortiz I, Nuñez E, Velasco E

Master in Implant Dentistry. Faculty of Dentistry. University of Seville.

\section{Abstract}

Introduction. Post extraction implants are an implantologic alternative to reduce treatment time and reduce atrophic changes after tooth extraction. The aim of this study was to show the results of treatment with dental implants insertec after extraction and immediately loaded.

Methods. 45 patients with unitary tooth loss were treated with 45 IPX (Galimplant $\left({ }^{\circledR}\right)$ internal connection, sandblasted and etched surface. implants. All implants were inserted after the corresponding extraction and were loaded immediately. At 3 months the definitive prosthesis were placed. No biomaterials or membranes were used.

Results. Clinical results indicate a survival and success rate of implants of $100 \% .60 \%$ of implants were inserted in the maxilla while $40 \%$ in the mandible. 45 resin provisional single crowns were placed immediately and ceramic restorations after 3 months. After a mean functioning period of 70.6 months, there were no complications

Conclusions. This study indicates that dental implants inserted in post-extraction and immediately loaded can be a predictable and successful implantologic alternative.

\section{Background and Aim}

The alveolar process is a specific tissue that develops together with the eruption of the teeth The volume of the alveolar process is related to the shape and size of the teethAfter a period of 6 months from tooth extraction, the mean horizonta reduction of the alveolar ridge is approximately 3.8 $\mathrm{mm}$ and $1.24 \mathrm{~mm}$ of vertical reduction.

To try to avoid these atrophic changes, we evaluate the possibility of inserting implants immediately after tooth extraction. The technique of immediate post extraction implants has been well documented in experimental animal studies showing that insertion of the implant immediately after tooth extraction does not totally prevent alveolar bone resorption, especially in the buccal wall. Histological findings in animals have shown that 2 more lingual position of the implant in the socket can reduce bone resorption and expose less the vestibular implant surface, being more importan than filling the gap with biomaterial and placing a membrane

The aim of this study was to assess the clinical outcomes of the immediate post extraction implants placement and immediate functional loading technique..$^{1-5}$

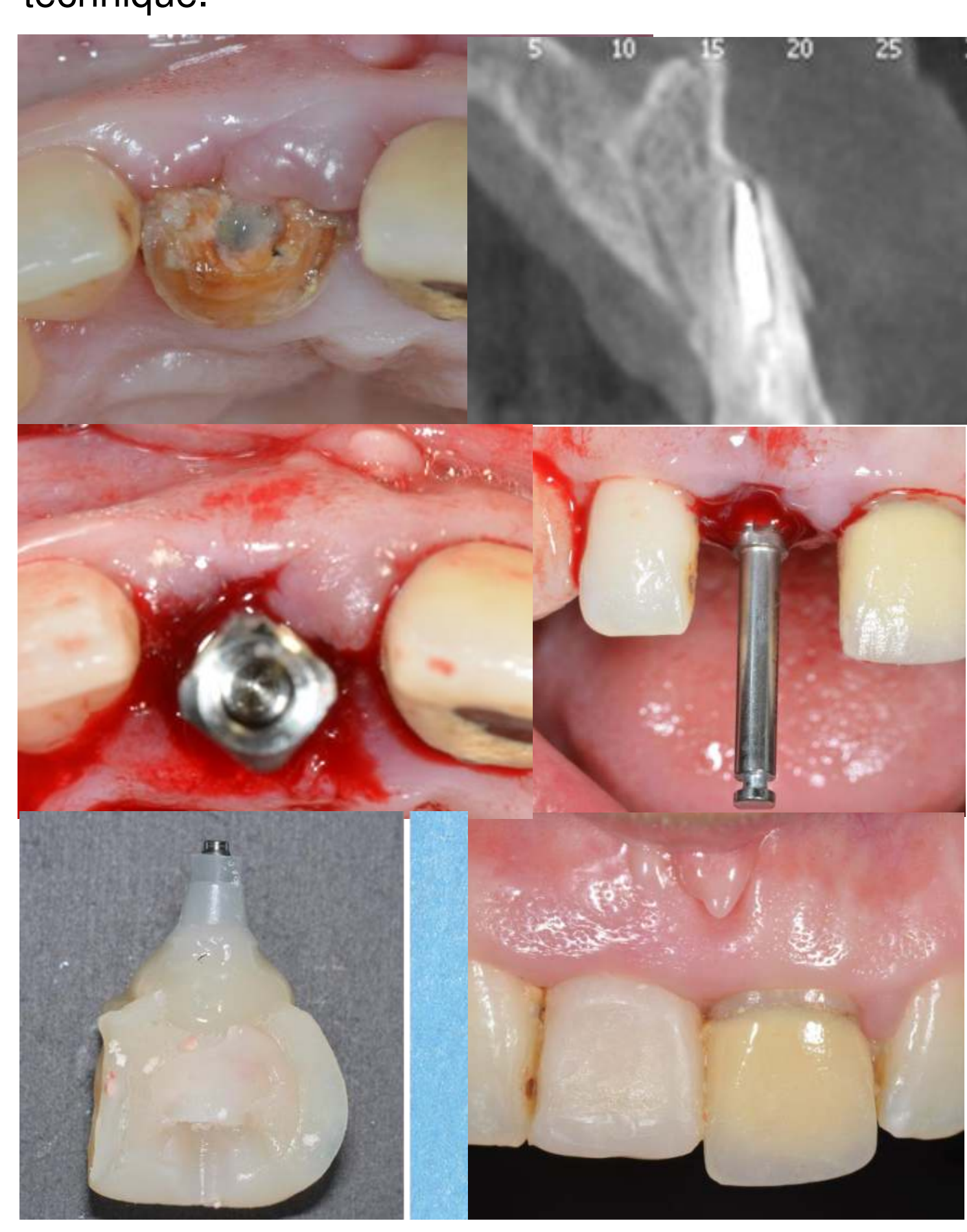

Figure 1. Postextraction and inmediate temporary restauration

\section{Methods and Materials}

This study was conducted in the Faculty of Dentistry at the University of Seville.

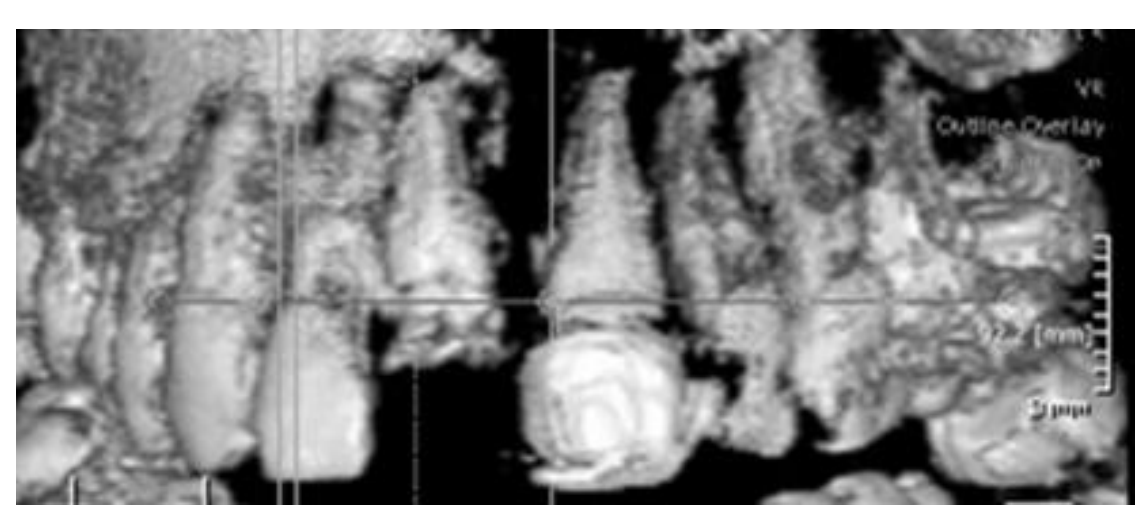

Figure 2. CBCT . 3D reconstruction

Prior to the study, patients with serious systemic disorders that could compromise osseointegration were excluded from the study. The criteria for implant success were those recommended by Albrektsson et al. ${ }^{6}$

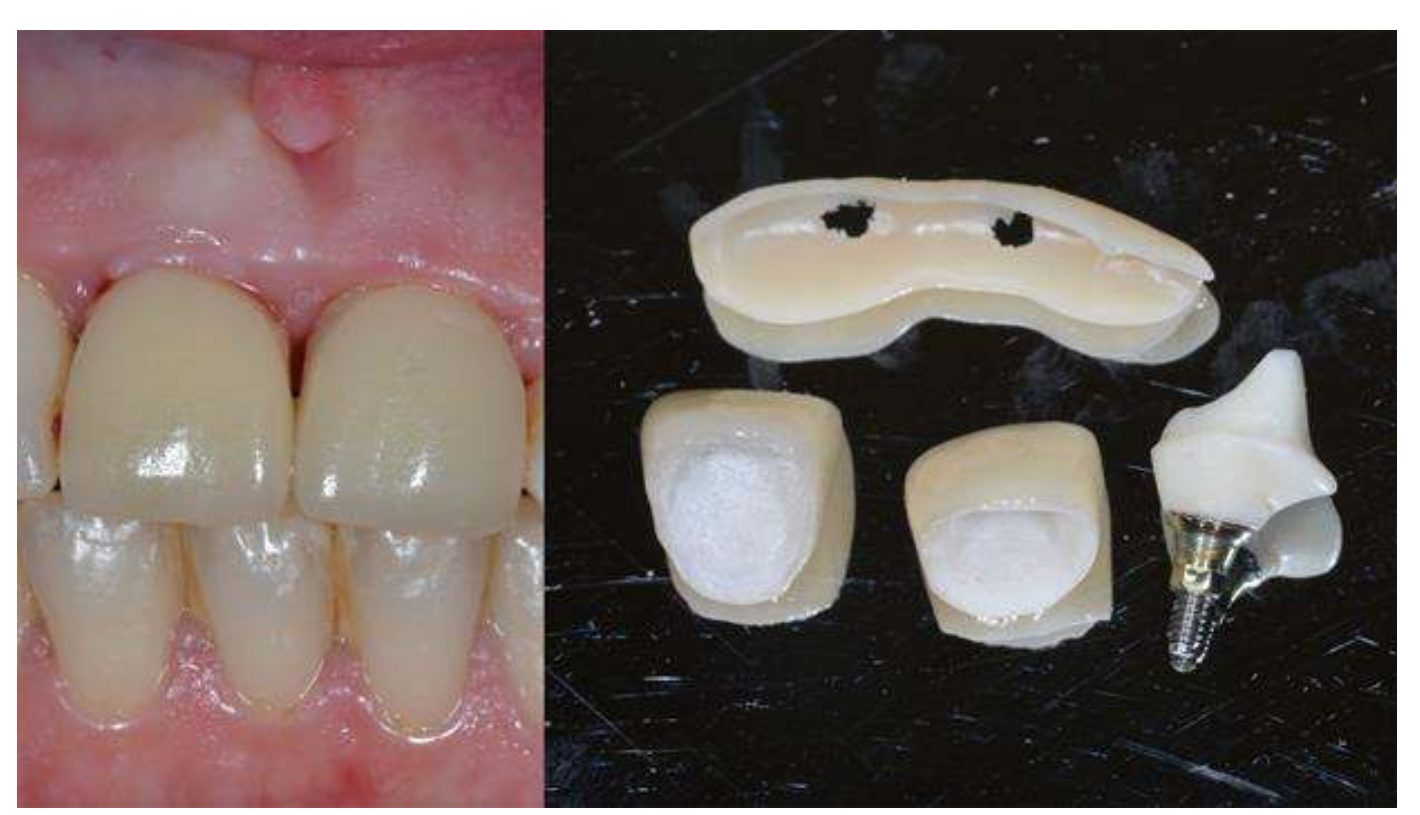

Figure 3. Definitive restauration after 3 months.

Surgery

All patients underwent a preventive antibiotic treatment. The immediate post-extraction implant technique consists in performing an atraumatic extraction with correct cleaning of the socket and then preform the preparation of the site $3 \mathrm{~mm}$ deeper than the apical limit of the socket in order to allow a good primary stability. The gap between the implant and the walls of the alveolus were not filled with any biomaterial.

Prosthesis

Inmediate loading protocol, placing the provisional restauration in the same surgical act, and the final prosthesis at 3 months, was established. The follow-up of functional loading was in average of 70,6months.. (Figs. 1-3).

\section{Results}

\section{Patients}

This study includes 45 patients with single or partial tooth loss, with an average age of 46,2 years (range 33-63). Graph 1

Graph 1. Gender distribution.

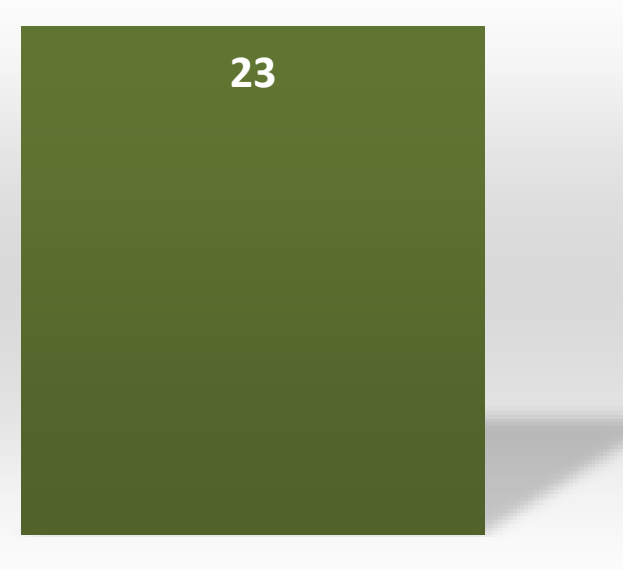
Male

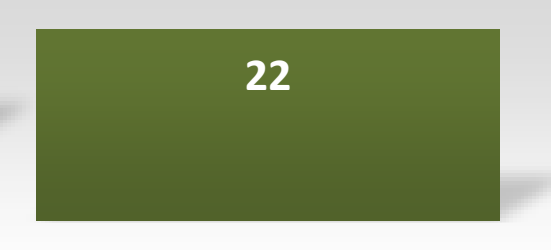

Implants

A total of 45 implants were inserted with diameters of: $3,5 \mathrm{~mm}$ (18 implants, $40 \%$ ); $4 \mathrm{~mm}$ (24 implants, $53,33 \%) ; 5 \mathrm{~mm}$ (3 implants, $11,11 \%)$ and regarding the length there were placed implants of: $10 \mathrm{~mm} \quad(6$ implants, $13,33 \%$ ); $12 \mathrm{~mm}$ (31 implants, $68,88 \%$ ); $14 \mathrm{~mm}$ (8 implants, 17,77 \%) (Graphs 2-5).

Graph 2-3. Diameter and length distribution.

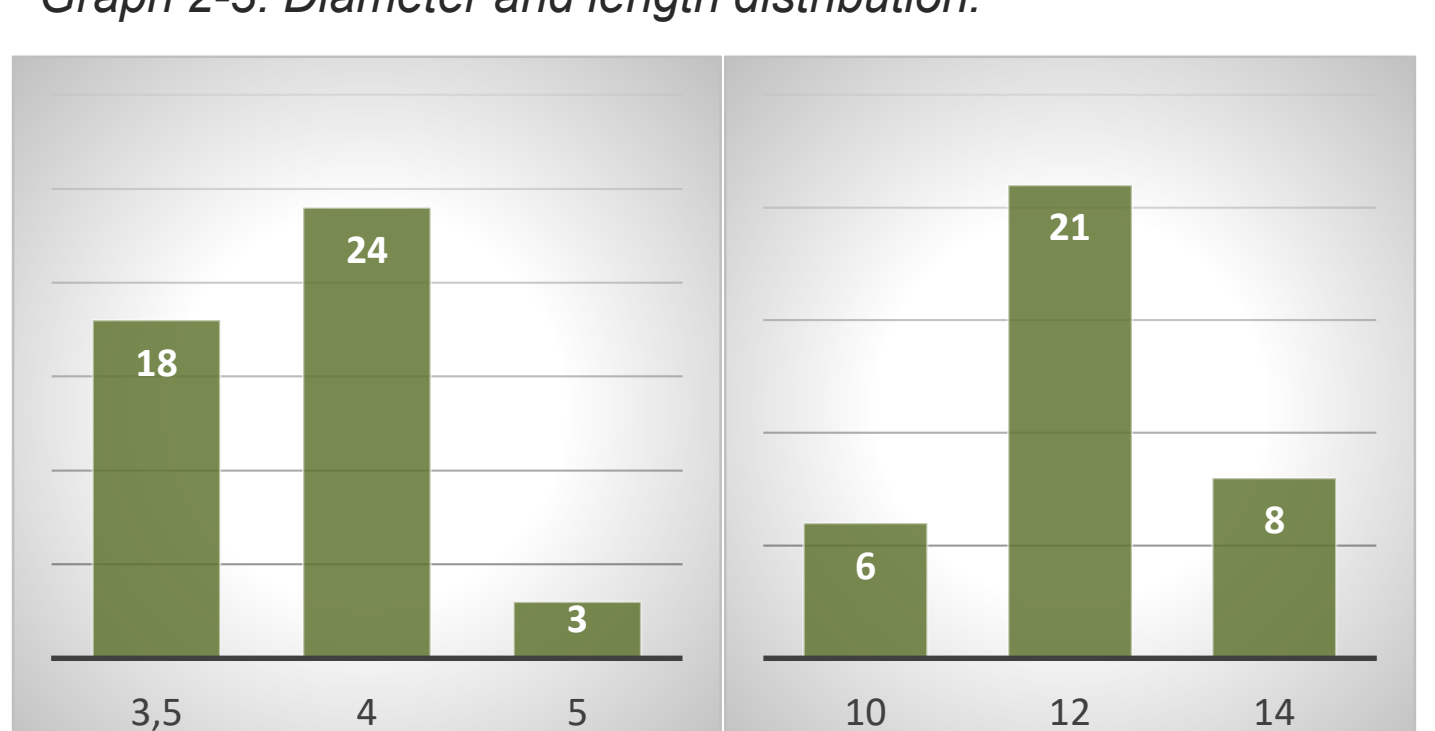

The average marginal bone loss was $0.67 \mathrm{~mm}$, with a range of 0 to $1.5 \mathrm{~mm}$. There were no surgical or prosthetic complications No implants were lost during the period of clinical follow-up.

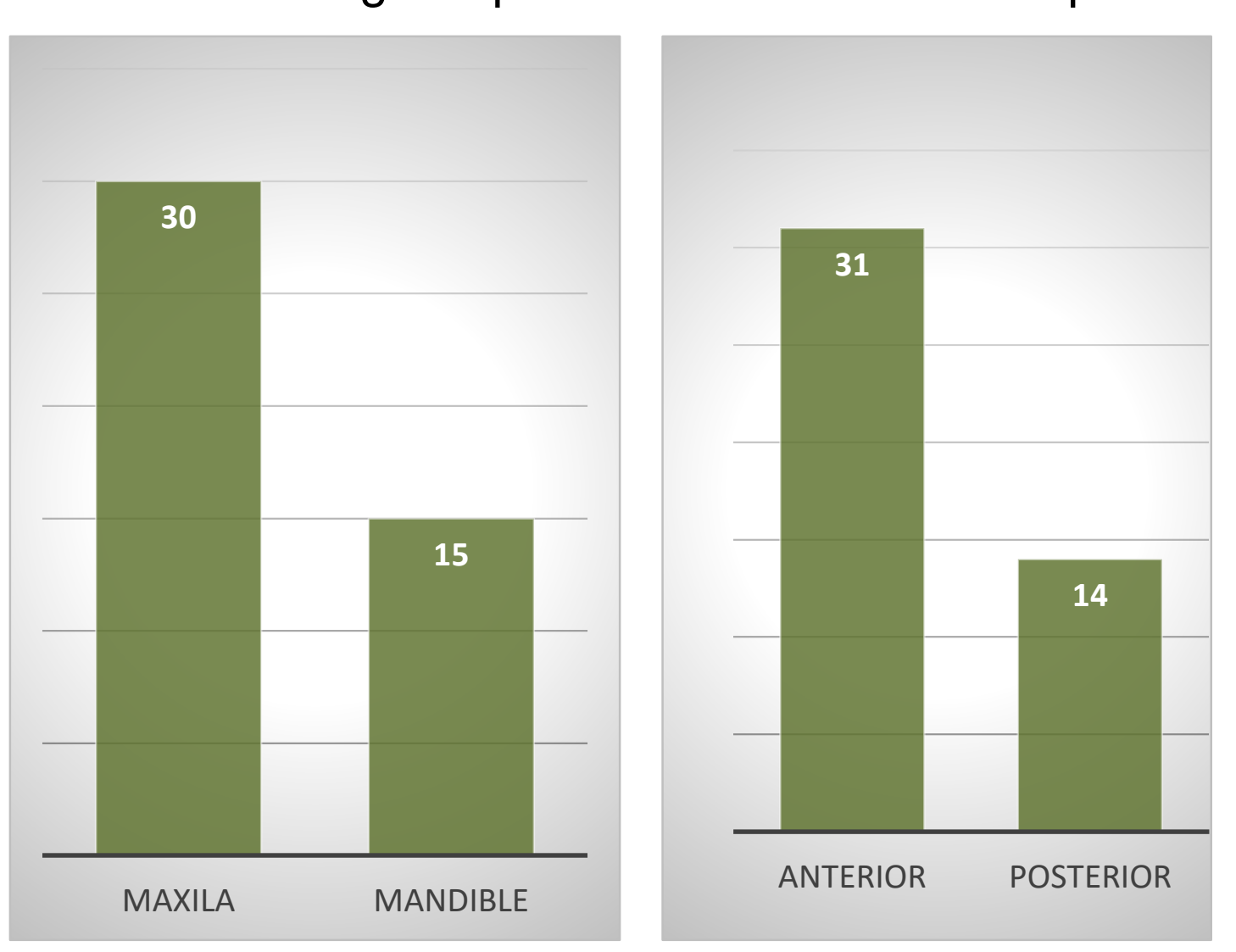

Graph 4-5. Jaw and area distribution.

Prostheses. 45 implants were immediately loaded with resin provisional prosthesis. At 3 months, the corresponding final ceramic restorations were made.

This study evaluates the clinical results of treatment with dental implants by inserting them immediately after extraction. This surgical technique has been gradually incorporated in the last 25 years with successful results

In the present study there weren't preformed any bone regeneration techniques with biomaterials or membranes, confirming that in cases of single post-extraction implants, these aren't essential to maintaining the peri-implant bone. ${ }^{8}$

The clinical results of this study indicates a high success rate of the immediate post-extraction implant technique $(100 \%)$ which concur with those reported by a recent review of the literature where 2,908 implants were evaluated in 46 studies with an overall success of $98,4 \%$ at 2 years. ${ }^{7}$

This study demonstrates the possibility of achieving osseointegration and stability of sandblasted-etched surface implants and when placed with the technique of immediate postextraction implants, macroscopic implant design is important for good primary stability during the surgical phase

Studies regarding immediate loading in immediate post extraction implants show that the success rate for these implants wouldn't differ if they had been inserted deferred after extraction or whether the loading protocol had been immediate, early or conventional. Overall bone loss was $0.6 \mathrm{~mm}$ per year of functional loading without differences between the two protocol.

\section{Conclusions}

Treatments with immediate post-extraction implant technique may represent a successful clinical alternative to help to prevent atrophic changes in the alveolar ridge. The performance of an adequate surgical technic can achieve excellent primary stability of the implant with functional and aesthetic restorations, maintaining a good level of peri-implant tissues.

\section{References}

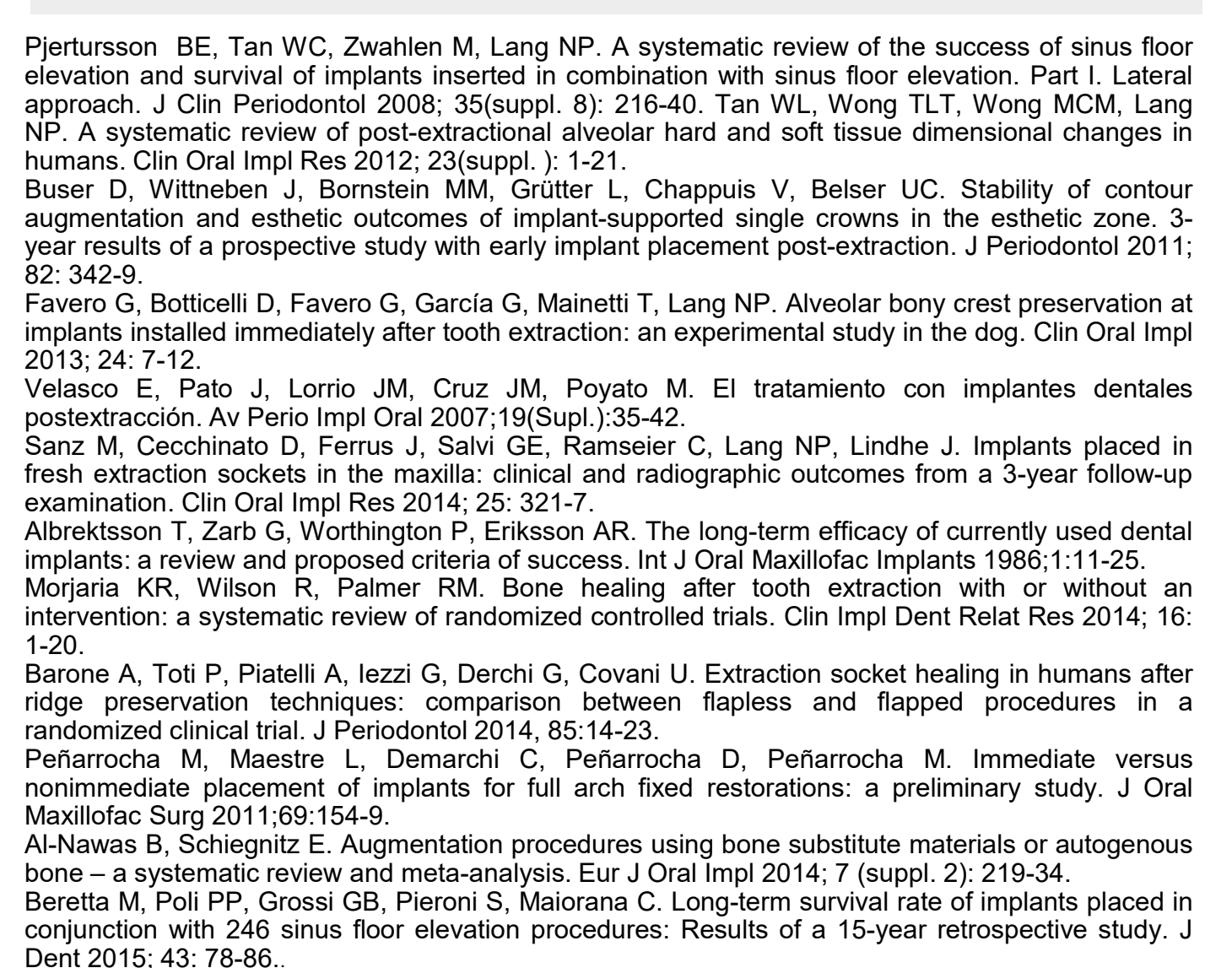

